\title{
The direct-current characteristics and surface repairing of a hydrogen-terminatedfree-standing polycrystalline diamond in aqueous solutions
}

Yuting Zheng ${ }^{\mathrm{a}, \mathrm{b}}$, JinlongLiu ${ }^{\mathrm{a}}$, JingiingWang ${ }^{\mathrm{c}}$, Zhengcheng Li ${ }^{\mathrm{d}}$, Hui Hao ${ }^{\mathrm{d}}$, Junjun Wei ${ }^{\mathrm{a}}$,Liangxian $\mathrm{Chen}^{\mathrm{a}}, \mathrm{Haitao}_{\mathrm{Ye}}^{\mathrm{b}}$, Chengming $\mathrm{Li}^{\mathrm{a}}$,

a. Institute for Advanced Materials and Technology, University of Science and Technology Beijing, Beijing 100083, PR China.

b. Department of Engineering, University of Leicester, Leicester, LE1 7RH,UK

c.National Key Laboratory of ASIC, Hebei Semiconductor Research Institute, Shijiazhuang 050051, PR China.

d. Suzhou Institute of Nano-Tech and Nano-Bionics(SINANO), Chinese Academy of Sciences, Jiangsu 215123,PR

China

E-mail address: ${ }^{a}$ nevermoreKEVIN@163.com, ${ }^{\text {byz516@leicester.ac.uk }}$

*Corresponding author

E-mail address: chengmli@mater.ustb.edu.cn, TEL: +8601062332390

Abstract:

As we know that more effective synthesis of diamond combined with physical and chemical properties of hydrogen termination in aqueous environment as well as device structure design can greatly facilitate the chemical and electrochemical applications of higher cost-performance diamond. For this purpose, the direct-current(DC)characteristics, surface reactionand reparation of a hydrogen-terminated DC arc jet plasma CVD polycrystalline diamond, which hasa high cost-performance, were characterized by $I-V$ experimentsbased on aFET-like structure device in multipleaqueoussolutions. The variation trends of the $I$ - $V$ properties of device based on $\mathrm{pH}$ were similar indifferent aqueous solutionsbut could be affected by disparate ions (such as $\mathrm{K}^{+}$ions)or organic molecules(such as citric acid radicalsora benzene ring).Especially, owing to the founded replacement of hydrogen termination with hydroxyl (-OH), carboxyl radical (-COOH)or carbon-oxygen bond (-C-O-C-) in mixed solution, i.e., $\mathrm{KHP}+\mathrm{H}_{2} \mathrm{SO}_{4}$ (and $\left.+\mathrm{NaOH}\right)$ solutions, the resistance of the device was remarkably increased from $13.57 \mathrm{k} \Omega$ to $95.78 \mathrm{k} \Omega$. However, the raised resistance of surface reacted diamond (SRD) can be reducedprominentlyby repairing hydrogenterminationthroughnegative potential sweeps (NPS)at a low negative potential (-1 to $-3 \mathrm{~V})$ ifthe SRDwas introduced as an electrode in a strong inorganic acid.What's more, the NPS repaireddevice, whichsubsequently stored for four weeks, wasmore sensitive than the original hydrogen plasma-treated diamond in aqueoussolution environments. This repaired resultwascoming out of NPS re-formed C-H bonds with higher intensity. These findings will be the references of failure and reparationof diamond hydrogen termination inaqueous environment.

Key Words:Direct-current properties, Hydrogen-terminated polycrystalline diamond, Negative potentialsweep, Surface repairing

\section{Introduction}

Diamond has been widely recognized as anultra-limit functional material as well as a promising material for next-generation semiconductor; this is due to its excellent intrinsic properties(such as having a high breakdown field, high thermal conductivity, high carriermobility, lowdielectric constant, and perfect performance in harsh environments, e.g.strong acid and alkali corrosion)[1-3]. Exhilaratingly, the quality of chemical vapor deposition (CVD) diamonds has been significantly optimized in recent years to the electronic-grade level[4]. Technologically, microwave plasma chemical vapor deposition (MPCVD) is the most widely usedequipment to synthesis SCD as well as $\mathrm{PCD}[3]$. Although the best option for the most demanding applications is single-crystal diamond (SCD), a high-quality polycrystalline diamond (PCD) is comparable - and even better in some situations. Meanwhile, one of the difficult issues in diamond technology is to manufacture large-size wafers. As mentioned, the problem ofenlarging the seed crystal, substrate and plasma volume are not so easy to resolve. The size of generally commercialized SCD wafersis still several millimeters(typically 2-3 mm at present); even PCD wafer is just 2-3 inch (around 50-80 mm). In this case, material size and limited production efficiencyas well as high price that really limits the application of 
diamond. Alternatively, acommercially available,high-quality,large-area, free-standing polycrystalline diamond with a high cost-performance can be prepared by a direct-current (DC) arc jet CVD technology with a high deposition rate; this can be done at a high cost-performance[5,6]. In particular, the larger and high cost-performance free-standing hydrogen-terminatedpolycrystalline diamonds(H-PCDs) can make it easier to fabricate electronic devices - such as $\mathrm{pH}$ sensors or detectors of ion concentrationsin extreme environments.

In terms of the application of hydrogen-terminated diamond semi-conductivity, the carriers channel has a lower activation energy than boron-doped ( $p$-type) diamonds[5]. Hydrogen termination cannot cause damage todiamonds; however, it does form $\mathrm{C}$-H bonds at the surface which induces $p$-type surface conductivity as a subsurface hole accumulation layer through the transfer of electrons[7,8]. The surface conductivity of a hydrogen-terminated diamond can be modulated by using an electrolyte gate between the surface of hydrogen terminated diamond and the aqueous solution; thismethod has recently resulted in several applications, e.g, ion sensitive field effect transistor (ISFET) $[8,9]$. In contrastto the surface charge transfer in inorganic solutions, organic molecules can also provide an acceptor, and the replacement of the terminated hydrogen atom byorganic groups have been investigated[10,11].However, there are a few systematic researches that investigating the electrical characteristic, surface $\mathrm{C}-\mathrm{H}$ bonds failure and surface reparation of hydrogen terminated diamond in multiple aqueous solutions. This information is very important for applications and our study covers this gap partially.

To the best of our knowledge, we used a high cost-performance DC are jet CVD free-standing polycrystalline diamond, whichsynthesized in large size (up to diameter $150 \mathrm{~mm}$ ) at high growth rate, to fabricate FET-like structure device using in aqueous environment for the first time. This kind of large size diamond wafer (or even can called plate) has been used for fabrication of electronic devices [5,7] and can be further utilized for more applications in chemical and electrochemical area. At the same time, we firstly found the surface $\mathrm{C}-\mathrm{H}$ damage in a certain solutionunder potential supply,however it could be recovered at low negative potential, which resulted in more stable and sensitive property of hydrogen terminated FET-like device in aqueous environment. Therefore, in order to avoid waste and reduce the cost of the diamond device, simple and non-scathed surface reparation method is a significant and meaningful way to re-produce and re-useahydrogen terminated diamond, which can degrade.

In this study,the trends of $I-V$ characteristicsof a hydrogen terminated DC arc jet CVD free-standing polycrystalline diamond based on $\mathrm{pHvaried}$ in different aqueous solutions. Surface reactionsthat increased the surface resistance in the $\mathrm{KHP}+\mathrm{NaOH}+\mathrm{H}_{2} \mathrm{SO}_{4}$ mixed solution, which contained a benzene ring were also observed. Furthermore, the raised surface resistances could be reduced by a negative potential sweep(NPS)process if the degraded diamond was used as the electrode in strong inorganic acidat a low negative potential. The recovered device was stableafter a period of storage time and more sensitive in an identical $\mathrm{H}_{2} \mathrm{SO}_{4}$ solution(compared to the original hydrogen plasma-treated device).Thesewillfurther promote the applications, e.g., ion concentration sensor, ion sensitive detector or electrochemical electrode, of diamond in aqueous environment, especially of which with higher cost-performance and higher production efficiency $[6,14]$.

\section{Experimental Methods}

Devices-used $15 \mathrm{~mm} \times 15 \mathrm{~mm} \times 0.7 \mathrm{~mm}$ polycrystalline diamond films were cut with a laser from a large free-standing polycry stalline diamond wafer, which had a diameter of $120 \mathrm{~mm}$. This high-quality, free-standing PCD wafer was deposited for $200 \mathrm{~h}$ on a titanium/graphite composite substrate at a deposition rate of $7.5 \mu \mathrm{m} / \mathrm{h}$ at 900-920 ${ }^{\circ} \mathrm{C}$, with an input power of $30 \mathrm{~kW}$ and a chamber pressure of 3.1-3.2 kPaby using home-built $100 \mathrm{~kW}$ DC arc jet plasma CVD technology. The plasma gas used was a mixture of argon and hydrogen, and the source gas used was methane. The $\mathrm{CH}_{4}$ concentration (volume percentage of $\mathrm{CH}_{4}$ in $\mathrm{H}_{2}$ flow rates) was varied in the range of 1-3.5\%.More synthetic technology details can be found at [12-15].The large-scale free-standing diamond wafer even 
could up to diameter of $150 \mathrm{~mm}$ or lager. This technology has opened the gate of larger diamond wafer production for thermal, optical or electrical use as well as higherefficient synthesis for more small size device application. Meanwhile, other aspects of cost reducing and high efficiency annealing by DC arc jet CVD process has been studied[6,14].After grinding and precision polishing, the film was cleaned in a boiling $\mathrm{H}_{2} \mathrm{SO}_{4}$ and $\mathrm{HNO}_{3}$ mixed acids for $1 \mathrm{~h}$ and then ultrasonically cleaned in acetone and methanol for decontamination.

The surface hydrogen termination was obtained by a microwave hydrogen plasma treatment under $800^{\circ} \mathrm{C}$ for $30 \mathrm{~min}$ in a home-built microwave plasma chemical vapor deposition (MPCVD)system with a 6-kW power supply (MUGGE, 2.45GHz and $6 \mathrm{~kW}$ ). Then, the FET-like structurediamond device was fabricated by using a lithography development process with a $150 \mathrm{~nm}$-thick Au layer as the source-like and drain-like electrodes, which were deposited at $50^{\circ} \mathrm{C}$ by electron-beam evaporation(EBE, Denton-Vacuum-Explore). The copper wires were connected to Au by using a silver conductive paste. All the metal parts were covered with silicone rubber (NANDA) to protect them from the chemical etching of thesolution environment. Au contacts were deposited as electrodes of hydrogen-terminated diamond metal-oxide-semiconductor field effect transistor (MOSFET)[2,16,17]. Theohmic contact properties of a $p$-type surface conductive layer on H-terminated diamond filmswhich had been prepared by DC arc jet CVDwere reported in depth in our previous work[17,18].For the as-deposited Au contact, the electricalpropertiesshowed a satisfiableohmic contact for electronic devices.

Multiple solvents have been chosen for preparing aqueous environment. The pure acid and alkali, acted as references to the other solutions which have same $\mathrm{pH}$, were used to verify the effect of disparate ions. Meanwhile, we employed them to realize the adjusting of $\mathrm{pH}$ of mixed solution as well as creation of more practical complex aqueous conditions.In detail, the solutions with various $\mathrm{pH}$ values (1 to 13) were prepared by using multiple solvent systems: $\quad \mathrm{H}_{2} \mathrm{SO}_{4}, \quad \mathrm{NaOH}, \quad \mathrm{KHP}\left(\mathrm{HOOCC}_{6} \mathrm{H}_{4} \mathrm{COOK}\right), \quad \mathrm{KS}\left(\mathrm{KHP}+\mathrm{H}_{2} \mathrm{SO}_{4}\right)$,

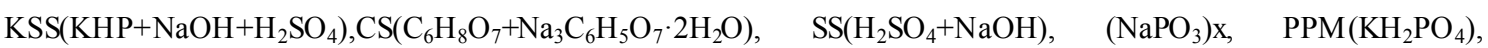
DHP $\left(\mathrm{Na}_{2} \mathrm{HPO}_{4} \cdot 7 \mathrm{H}_{2} \mathrm{O}\right), \mathrm{NaHCO}_{3}+\mathrm{NaOH}, \mathrm{Na}_{2} \mathrm{HPO}_{4}+\mathrm{NaOH}$, and $\mathrm{Na}_{2} \mathrm{~B}_{4} \mathrm{O}_{7} \cdot 10 \mathrm{H}_{2} \mathrm{O}$. Therefore, the DC characteristic of hydrogen terminated FET-like structure diamond device in multiple aqueous solutions can be systematically investigated and the surface $\mathrm{C}-\mathrm{H}$ bonds stability in these solutions under potential supply can be evaluated as well. The DC properties of the FET-like diamond devices were investigated by using an electrochemical workstation(CHI660E) in disparate aqueous solutions. During the testing process, the intact devicewas immersed into multiple solvents aqueous environmentin an electrolytic tank, and two copper wires were connected to the working electrode and counter electrode respectively. A pH electronic tester (PIPH-20,accuracy is 0.01) was used for the $\mathrm{pH}$ measurement of diverse solutions after being calibrated by the standard solution. In addition, the resistances were measured by using an ultra-precision smart multimeter(UT204A, accuracy is $0.001 \Omega$ ).

At the same time, XRD characterization (Rigaku TTR), using $\mathrm{CuK}_{\alpha l}$ radiation $(\lambda=1.54056 \mathrm{~nm})$ with an incident X-ray angle of $10^{\circ}$ was employed to investigate the different crystal orientations of polycry stalline diamond. A visible Raman spectroscopy (HORIBA Scientific) measurements with a 532-nm excitation wavelength was used to examine the quality of diamond. The surface morphologies of the treated diamond surfaces were compared by using an atomic force microscope(AFM, ASYLUM RESEARCH HVA220). Owing to the limitation of $\mathrm{H}$ ion detection, wetechnicallyemployed the time-of-flight secondary ion mass spectroscopy (TOF-SIMS, IONTOF, GmbH, TOF-SIMS-5 generation), which is the latest generation of high-end TOF-SIMS instruments and with ultra-high resolution[19],to sensitivelydetect plasma created surface $\mathrm{H}$ terminationions andstraightforwardlydisplay the ion distribution as a function of incident ion sputter time. The incident ion was $\mathrm{Cs}^{+}$, which irradiated bulk diamond from surface to inner-body at $2 \mathrm{keV}$ incident energy. After that, in order to determine the organic group and $\mathrm{H}$ termination replacement on the diamond surface, Fourier transform infrared spectroscopy(FTIR spectrometers, BRUKER, ALPHA-II,) was employed, and the attenuatedtotalreflectionFTIR(BRUKER, LUMOS, ATR-FTIR 
spectrometers)was utilized to detect NPS re-formed C-H bonding on the diamond surface. A Ge crystal was used as the materialfortotal reflection. The angle of incidence was $45^{\circ}$, and a measuring resolution of $4 \mathrm{~cm}^{-1}$ was chosen. Meanwhile, owing to the inevitablesolv entresidue after operation in aqueous solutions, which could affect TOF-SIMS test results of $\mathrm{H}$ termination, the subsequent FTIR and ATR-FTIR were the appropriate way to investigate the surface reaction. Of course, the ATR-FTIR spectrum of the plasma treated sample,which has been tested by TOF-SIMS, will be presentedas a reference to prove the $\mathrm{C}-\mathrm{H}$ replacement andreparation.

\section{Results and Discussion}
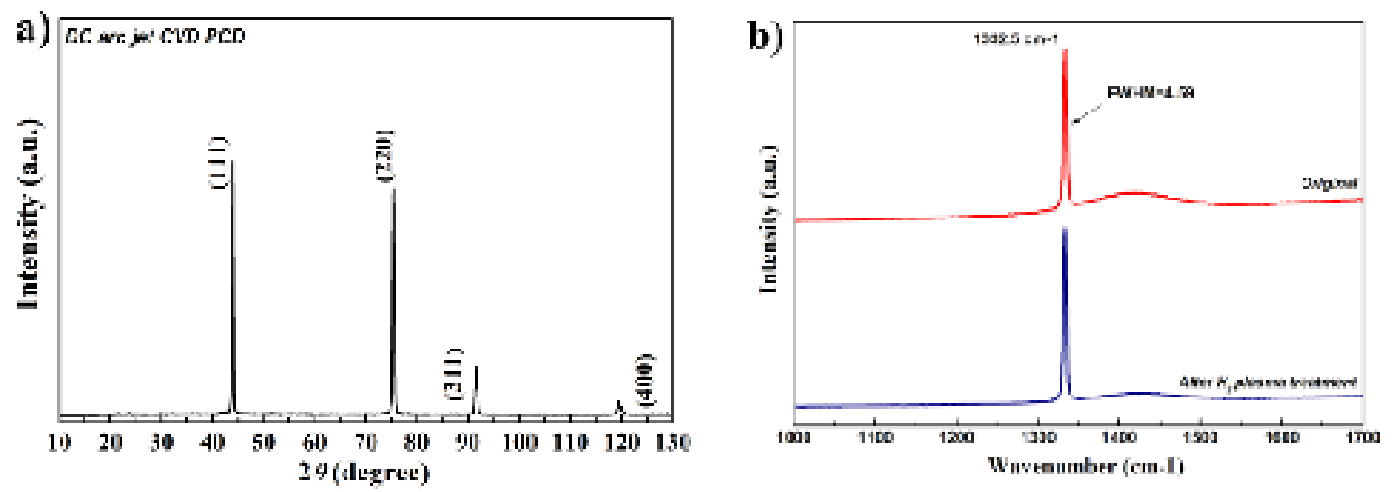

Fig.1 Structure and phase quality characterization of synthesised PCD: a) XRD spectrum of original PCD and b) Raman spectra of PCD before and after hydrogen plasma treatment

The use of XRD is necessary when investigating poly-crystallographic orientation of PCD and the optimal orientation and crystallinity can be judged as well. Fig.1(a) shows the XRD of the original PCD sample which was introduced to fabricate FET-like structure device. An example of the diverse crystal orientations in polycrystalline materials, the original DC arc jet plasma PCD consists of (111), (110), (311) and (400) orientations and its preferential orientation are (111) and (110)[20]. The high quality of diamond crystal can be judged by the sharp and high intensity XRD peaks in Fig.1(a) as well as Raman spectrum. As shown in Fig.1(b), the displayed single first-orderRaman characteristic line of $1332.5 \mathrm{~cm}^{-1}$ was prominentand no any other non-diamond peak in the range from $1000-1700 \mathrm{~cm}^{-1}$. At the same time, the full width at half maximum (FWHM) of $s p^{3}$ diamond Raman characteristic line was $4.59 \mathrm{~cm}^{-1}$ (based on the Gaussian fitting) and lower background of spectrum both indicate that the crystal quality was excellent. Owing to the grain boundary and it caused inner-stress, the theoretical diamond characteristic Raman peak of $1332 \mathrm{~cm}^{-1}$ was slightly shifted to $1332.5 \mathrm{~cm}^{-1}$ [12]. Of course, there is an apparent broad band at around $1430 \mathrm{~cm}^{-1}$ in Raman spectra, this was resulted from mechanical polishing caused surface defects[21]. However, thesesurface defects, i.e., undesired disorder or damage, can be etched by plasma treatment.As shown in Fig.1(b), $1430 \mathrm{~cm}^{-1}$ broad band was weaker after hydrogen plasma treatment[22]. This process will be mentioned in the following parts and more detailed investigation regarding this kindofdefects related band weaken effect by plasma etching will be presented in our other literature soon. 

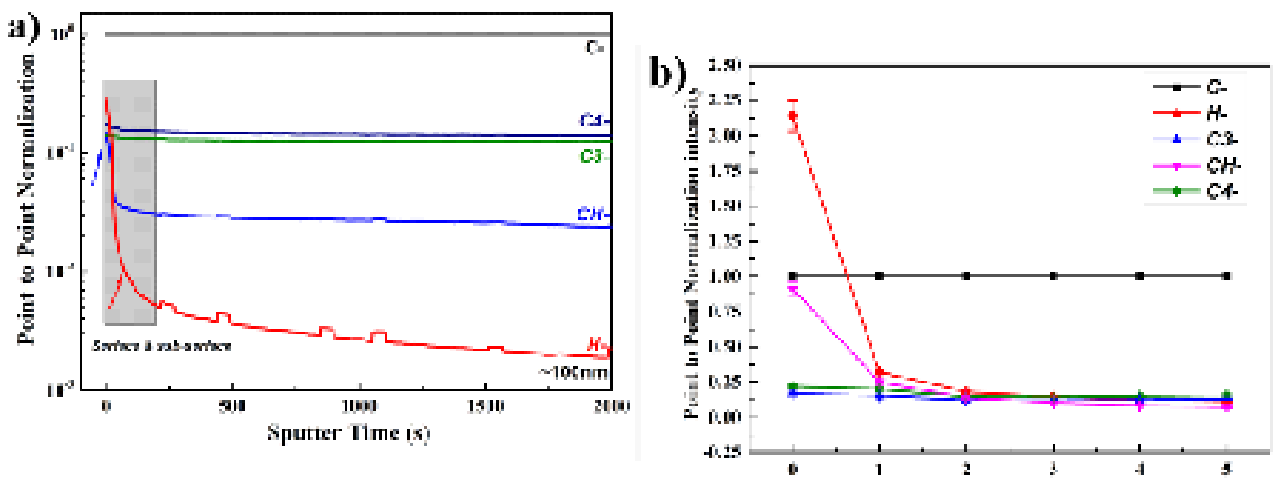

Fig. 2 TOF-SIMS spectra of ion distribution as a function of sputtertime: a) sputter time of from 1 to $2000 \mathrm{~s}, \mathrm{~b}$ ) surfacefine ion intensity as a function of sputter time of from 0 to $5 \mathrm{~s}$

As whole investigation was depended on hydrogen termination, thus surface characterization techniques must be employed to verify the surface hydrogen termination. We adopt TOF-SIMS, the most sensitive surface analysis (even including light element of hydrogen) technique[19], to examine the surface plasma formed hydrogen termination. Fig. 2 (a) shows the ion distribution as a function of incident ion sputtertime, the data of detected $\mathrm{C}$ and $\mathrm{H}$ elements have been point-to-point normalized. Owing to the overwhelming amount of surface $\mathrm{H}$ ions and thus in order to better display the distribution of $\mathrm{H}$ and $\mathrm{C}$ ions in bulk PCD, we set the sputter time of spectra of Fig.2(a) was from 1s to 2000s. Of course, the finelocal intensity of surface $\mathrm{C}$ and $\mathrm{H}$ ions is shown in Fig.2(b) to examine the surface hydrogen termination in microscale (sputter time was just from 0-5s). As shown in Fig.2(a), the secondary ion contents of $\mathrm{H}$ - and $\mathrm{CH}$ - were drastically declined with the sputter time of $\mathrm{Cs}^{+}$beam going by, which indicates the $\mathrm{H}$ element was significantly decreased from diamond surface to inner-body. The reason why some $\mathrm{H}$ were remained in bulk diamond crystal is that the diamond was synthesized inrich hydrogenplasma environment and especially the hydrogen termination could be formed on the surface of crystal grains inside PCD. As we all know that SIMS is generally considered to be a qualitative technique, although quantitation is possible with the use of standards[23]. In Fig.2(b), the C- was normalized to be 1 as reference to other ions or atomic group. Therefore, the $\mathrm{H}$ termination ions proportion $r_{\mathrm{H}}$ can be calculated as the equation (1) we set as below:

$$
r_{\mathrm{H}}=\frac{C_{\mathrm{H}-}+C_{\mathrm{CH}-}-2 C_{\mathrm{O}-}}{C_{\mathrm{C}-}+3 C_{\mathrm{C} 3-}+4 C_{\mathrm{C} 4-}+C_{\mathrm{CH}-}}
$$

Where $C$ is the content of ions or atomic group, e.g., $\mathrm{H}^{-}, \mathrm{C}_{3}^{-}$, which are shown as right subscript. The error range of the values we set was 5\% which are presented as error bar in Fig.2(b). Here should be note that tiny moisture on diamond surface during testing must be taken into consideration. Therefore, we subtracted the $2 \times C_{0}$ which comes from $\mathrm{H}_{2} \mathrm{O}$ of moisture in equation (1). The $\mathrm{H}$ termination proportion to $\mathrm{C}$ ions of diamond was $87.69 \pm 4.4 \%$. This value was not as high as $100 \%$ was maybe due to the surface defects, grain boundary or different crystal orientation of PCD. In addition, the TOF-SIMS measured sample was tested by ATR-FTIR technique as well, which was acted as reference to other reacted samples in multiple aqueous solutions. Owing to the inevitablesolventresidue after operation in aqueous solutions, which could affectTOF-SIMS test results of $\mathrm{H}$ termination, the subsequent FTIR and ATR-FTIR were introduced to appropriately investigate and verify the surface reaction, which will be discussed in the following section. 


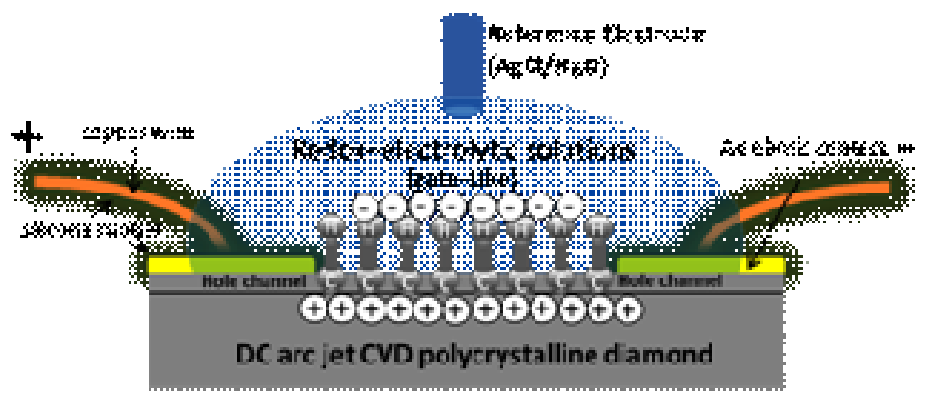

Fig. 3 The schematic of a FET-like structure H-PCD device in a redox-electrolytic solution.

Fig. 3 shows the schematic diagram of the hydrogen terminated diamond FET-like structuredevice in a redox-electrolytic solution. Technically, the reference electrode was introduced ( $\mathrm{AgCl}$ for acidic and neutral solutions, $\mathrm{HgO}$ for alkaline solutions) to accurately determine the potential of the working electrode during the measurement process. The resistances between the copper wire and the diamond surface of FET-like structure device samples were 67.3 86.5 $\Omega$. The DC characteristics of these devices were measured by using an ultra-precision smart multi-meter between one copper wire terminal and a specific point of the diamond surface; this was done in an atmospheric environment. The contact resistances of the sample devices (between copper wire and diamond surface) were approximately three orders of magnitude less than those of two copper wire terminals, whichincludes the resistance of naked hydrogen termination area, in the same situation. Theoretically, the presence of the oxidative ingredient as the electron accepter could significantly increase the degree of electron transfer between the C-H surface/solution; thus, this situation that hydrogen terminated area of diamond surface contact with oxidizing solution can be acted as the gateelectrode for this FET-like structure once this device has been immersed in an aqueous solution[9]. Due to the accumulation of holes introduced by electron movement, as shown in Fig. 3, a hole channelformed at the subsurface of the diamond. Therefore, the current could be detected if the potential difference was supplied between two copper wires.
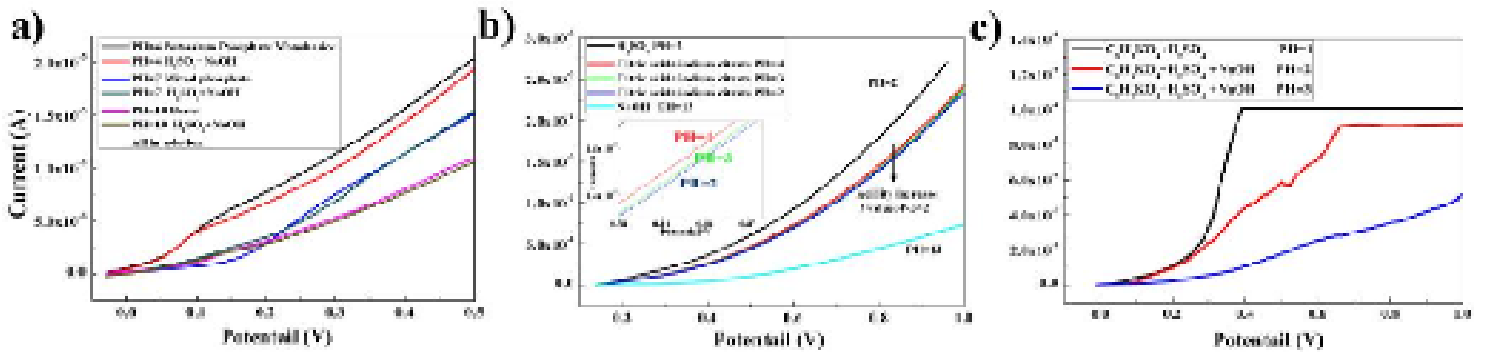

Fig. 4.DCcharacteristics of the device in different aqueous solutions: a) in diverse solutions with pH of 4, 7 , and 10 compared with the $\mathrm{H}_{2} \mathrm{SO}_{4}+\mathrm{NaOH}$ mixed regulating solutions; b) in $\mathrm{CS}$ solutions with $\mathrm{pH}$ of 2,3 , and 4 compared with pure acid $\left(\mathrm{H}_{2} \mathrm{SO}_{4}\right)$ and alkaline solutions $(\mathrm{NaOH})$; and c) in $\mathrm{KS}(\mathrm{KSS}: \mathrm{KS}+\mathrm{NaOH})$ solutions.

Fig.4 shows the $I-V$ patterns of DCcharacteristics of the device with the same linearly increased source-like and drain-like electrodes' potential differences in various aqueous solutions. Fig. 4(a) shows the $I$ - $V$ characteristics of the device immersed in diverse inorganic acid solutions; the current increased as the $\mathrm{pH}$ dropped. The current value in the PPM solution was slightly more than that in the SS solution, which had a pH of 4.This may have been due to the additional $\mathrm{K}^{+}$positive ions, which attract electrons. However, the result in the inorganic acid solution (such as mixed phosphate or borax) was almost identical to the data of the SS solvent system, which wasresulted from the same amount of $\mathrm{H}^{+}$(same $\mathrm{pH}$ value) in these solutions and did not have other positive ions which could attract electrons. The polylinesappeared at the beginning of the curve during the initial measurement due to the grain boundary of the polycrystalline diamond; however, the polylines disappeared after being activated by testing several times. In Fig. 
4(b), the changing rule of the $I-V$ characteristicsthat shows different in acid and alkali solution such as $\mathrm{H}_{2} \mathrm{SO}_{4}$ and $\mathrm{NaOH}$ solutions, which werebased on the $\mathrm{pH}$, was the same as that in Fig. 4(a). However, the $I$-V characteristics of the device in the $\mathrm{CS}$ solution were enhanced as the $\mathrm{pH}$ increased, which was op posite to the DC characteristic of H-PCD in inorganic aqueous solutions (from apH of 2 of a $\mathrm{H}_{2} \mathrm{SO}_{4}$ solution to a pH of 13 of a $\mathrm{NaOH}$ solution). However, there was no large difference among the $I-V$ data of characteristics of the device in CS-mixed solutions (that had a pH of 2 to 4).As a matter of fact,citric acid is an organic weak acid, ortricarboxylic acid compounds, which hasthree $\mathrm{H}^{+}$that can be ionized. Theseparate order ionization constant of citric acid is $p k_{1}=3.13, p k_{2}=4.76, p k_{3}=6.40$, which corresp onding to the first, second and third order ionization, respectively.Thus, citric acid is relatively acidic from the perspective of ionization constant.Most of ionized ion are hydrogen ions, and the main form of citric acid after ionization is related to $\mathrm{pH}$.The following chemical formulas are the first, second and third order ionization in aqueous environment:

$$
\begin{aligned}
& \mathrm{H} 3 \mathrm{Cit}==\mathrm{H}^{+}+\mathrm{H} 2 \mathrm{Cit}^{-}\left(p k_{1}=3.13\right) \\
& \mathrm{H} 2 \mathrm{Cit}^{-}==\mathrm{H}^{+}+\mathrm{HCit}^{-}\left(p k_{2}=4.76\right) \\
& \mathrm{HCit}^{-}==\mathrm{H}^{+}+\mathrm{Cit}^{-}\left(p k_{3}=6.40\right)
\end{aligned}
$$

As the $\mathrm{pH}$ value decreased, more and more citric acid radicals were ionized and vice versa. Therefore, the two $I-V$ curves of the FET-like device in solutions (which were tested in $\mathrm{H}_{2} \mathrm{SO}_{4}$ and CS solutions, respectively,and both had apH of 2)wereapparently different; this wasbecause of the citric acid radicals. However, although more citric acid radicals can counteract the variation trend, which depends on lower $\mathrm{pH}$ value, the results demonstrate that differences in the amount of citric acid radicals did not significantly change in the CS-mixed solutions (raising the $\mathrm{pH}$ from 2 to 4).Fig. 4(c) shows the $I-V$ characteristics of the device in KS and KSS (with the addition of $\mathrm{NaOH}$ ) solutions with a $\mathrm{pH}$ of 1,2 , or 3 . Potassium hydrogen phthalate (KHP) is a strong alkali and weak acid salt that can produce hydroxide ions by hydrolysis and can also ionize hydrogen. It can be used as a buffer substanceto react with acids or alkali solutions. Therefore, in a KS solution with a pH of 1, all the hydroxide ions of KHP werebroken away, and the remaining host radical group (whichhas intensive oxidizing ability) will strongly attract electrons from the hydrogen-terminated surface ofthe device if it comes in contact with an aqueous solution. Thus, the current of the device drastically increased to a plateau of $1.007 \times 10^{-4} \mathrm{~A}$ in the $\mathrm{KS}$ solution with $\mathrm{pH}$ of 1 . Adding $\mathrm{NaOH}$ to this mixed solution changed the $\mathrm{pH}$ value to 2 . The maximal current was $9.12 \times 10^{-5} \mathrm{~A}$, and the sharp fold line during the potential increasing process indicates that a surface reactionhad emerged. Notably, the number of holes, which resulted from the electron transfer, reached the limitation of the surface-effective $\mathrm{C}-\mathrm{H}$ amount of hydrogen terminated diamond, whichcaused the horizontal line of the current. In the KSS (KSand $\mathrm{NaOH}-$ mixed) solution with a pH of 3, the performance of this device degraded remarkably to $4.5 \times 10^{-5} \mathrm{~A}$ when a potential difference between source-like and drain-like electrodes was $1 \mathrm{~V}$ and a little bit of undulation appeared. 


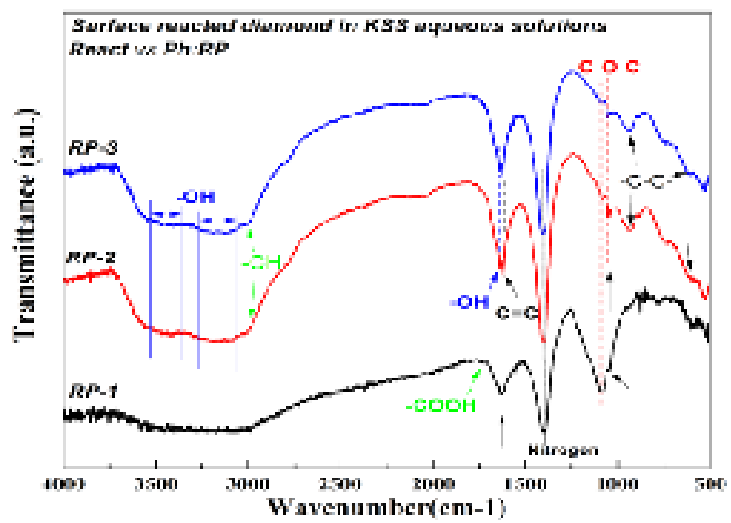

Fig.5The FTIR spectra of PCD films for the FET-like device that reacted in the KS(KSS) solution.

FTIR is a technique used to obtain an infrared spectrum of absorption or the emission of solid. It collects high-spectral resolution data over a wide spectral range to reflect the type of functional groups and the chemical environment where the diamond is contained.Fig. 5 shows theFTIR spectra of the PCD of a device that reacted in a $\mathrm{KS}(\mathrm{KSS})$ solution, correspondingto the $I-V$ results of Fig 4(c). It is important to note that the -OH bonds peak at 3300 - 3500 and that $1620-1630 \mathrm{~cm}^{-1}$ were related to adsorbed water, which provides strong absorption bands[16]. RP-1 sample, which was tested in a KS solution without $\mathrm{NaOH}$ being adding, the spectra had almost none of the absorption peaks of the -OH group. However, compared with the RP-1 sample, the peaks of the -OH group exist in the spectrum of RP-2 and RP-3, which were reacted in mixed KSS solutions; this maybe due to the-OH replacing reaction in a KSS solution with $\mathrm{NaOH}$ addition. Likewise, some explanations of absorbed water peaks are presented so that the diamond's peaks also exist in a vacuum after the desorption of water. The strongest evidence that the hydroxylation reaction took place was the presence of a band of around $3100-3200 \mathrm{~cm}^{-1}$ and $3400-3500 \mathrm{~cm}^{-1}$ (stretch vibration of the -OH group)[24]. Meanwhile, peaks within the $1000-1200 \mathrm{~cm}^{-1}$ region can be assigned to C-O-C vibrations in alcohol groups[24,25]. Herein, these group peaks (1100 and $\left.1045 \mathrm{~cm}^{-1}\right)$ in Fig.5 are sharp, but their density decreased from RP-1 to RP-3,reacting in the KSS solution with different $\mathrm{pH}$ values.Regarding the RP-1 sample, which reacted in the KS solution without $\mathrm{NaOH}$, the $-\mathrm{COOH}$ weak peak was at $1795 \mathrm{~cm}^{-1}$ in Fig.5[24]. However, these peaks of RP-2 and RP-3 (which reacted in the KSS solution) disappeared with the addition of NaOH. Above all, this demonstrates that the $\mathrm{C}-\mathrm{H}$ surface(which was produced by hydrogen plasma)was replaced by different organic groups in the KS and KSS solutions when potentialdifference wassupplied between source-like and drain-like electrodes. A strong band at $1570 \mathrm{~cm}^{-1}$ occurred. This peak can beattributed to other functionalities on the carbon surface, such asamide-related bands or stretching vibrations of aromatic $\mathrm{C}=\mathrm{Cbonds}$, which were polarized by oxy gen atoms bound near one ofthe carbon atoms[26].Additionally, the absorption peak at $1457 \mathrm{~cm}^{-1}$ was related to the nitrogen of the diamond sample[27].

Table 1 Resistances of the device after the surface reaction and NPS.

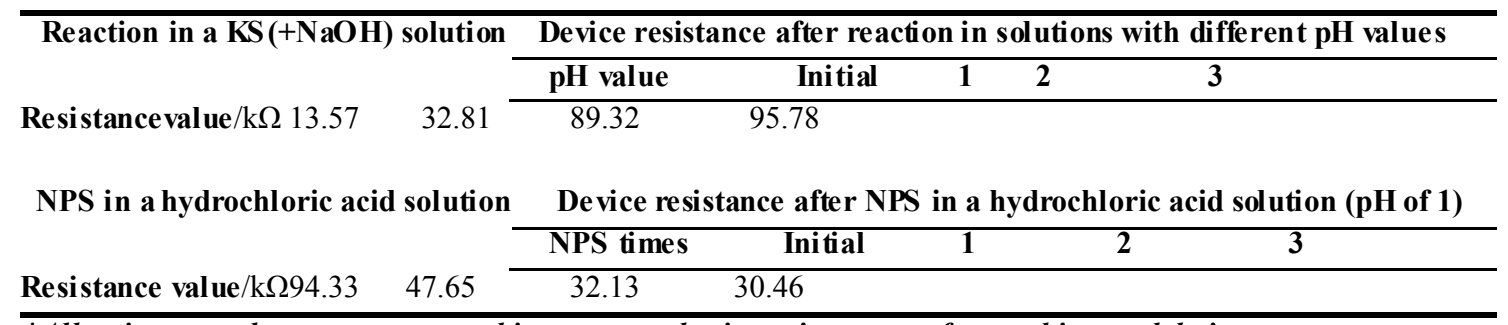

*All resistance values were measu red in an atmospheric environment after washing and drying.

Table 1 shows that the resistances of the device increased from the initial stage(before the test) at $13.5 \mathrm{k} \Omega$ to 
$32.8 \mathrm{k} \Omega$ after the first measurement in the KS solution (which had a pH of 1). The resistances drastically increased to $89.32 \mathrm{k} \Omega$ after being tested in the KSS solution (which had a pH of 2) and finally to $95.78 \mathrm{k} \Omega$ after being tested in the KSS solution (which had a pH of 3). Thus,some surface C-H bonds were probably damaged when the potential was applied in a specific aqueousenvironment(which was the K SS solutionin this case). In return, the current value of the device obviously decreased when it was examined in the KSS solution (which had a pH of 3 ). As shown in table 1, corresponding to the curve in Fig. 6, the resistances decreased from $94.33 \mathrm{k} \Omega$ to $47.6 \mathrm{k} \Omega$, then to $32.1 \mathrm{k} \Omega$, and eventually to about $30 \mathrm{k} \Omega$. All of these resistance values were measured in an atmospheric environment after washing and drying. Additionally,strong inorganic acid solutions (such as $\mathrm{H}_{2} \mathrm{SO}_{4}$ with $\mathrm{pH}$ values of 1 - 3) were also achievable for the NPS, which had been investigated by us.

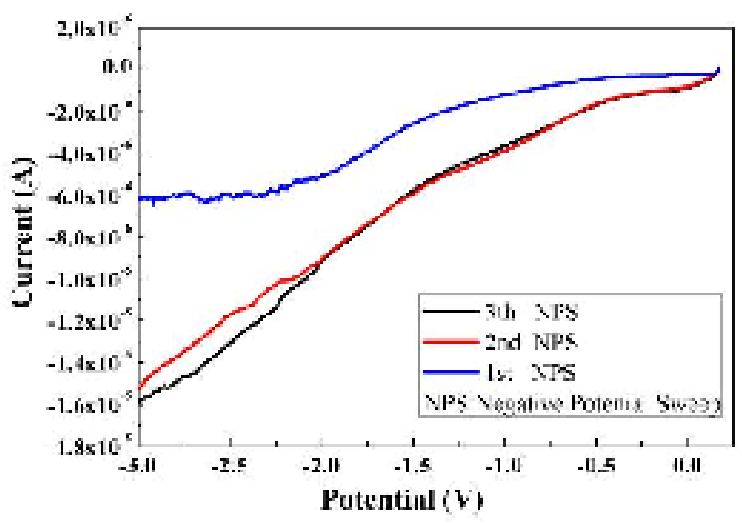

Fig.6 The I-V characteristics of the surface reacted device (SRD), which as an electrode in inorganic acid,during negative potential sweep (NPS).

Fig. 6 shows the $I-V$ curves of the surface reacted device (SRD) electrode's NPS process, whichintroduced a whole SRD as the working electrode (two copper wires of the device electrode were connected to a working electrode of an electrochemical workstation) in an inorganic acid solution with a $\mathrm{pH}$ of 1 . It should be mentioned that the solution had $0.1 \mathrm{M}$ of $\mathrm{HCl}$ with a $\mathrm{pH}$ of approximately 1 . During the process of relatively increasing the lower potential between the working electrode and the counter electrode of NPS from $-2 \mathrm{~V}$ to $-3 \mathrm{~V}$, the current of the SRD electrode reacheda plateauof around $-6.0 \times 10^{-6} \mathrm{~A}$ and fluctuated on a small scale, indicating that the damaged hydrogen termination had been repaired.A large amount of $\mathrm{H}^{+}$ions reached the diamond surface, resulting in the negative potential to form $\mathrm{C}-\mathrm{H}$ bonds with $\mathrm{C}$ atoms; thisbrought about electrons transferring easily to $\mathrm{HCl}$ solution and moving to counter electrode which hold a positive potential in the electrochemical system. Then, the constant currentdisappeared in the second and third NPS processes with the potential changing, which means that more electrons easily moved from the diamond surface to the solution environment thanks to the re-formed more $\mathrm{C}-\mathrm{H}$ bonds and that the $I-V$ curve only hadtiny fluctuations and increased to approximately $-1.6 \times 10^{-5} \mathrm{~A}$. 


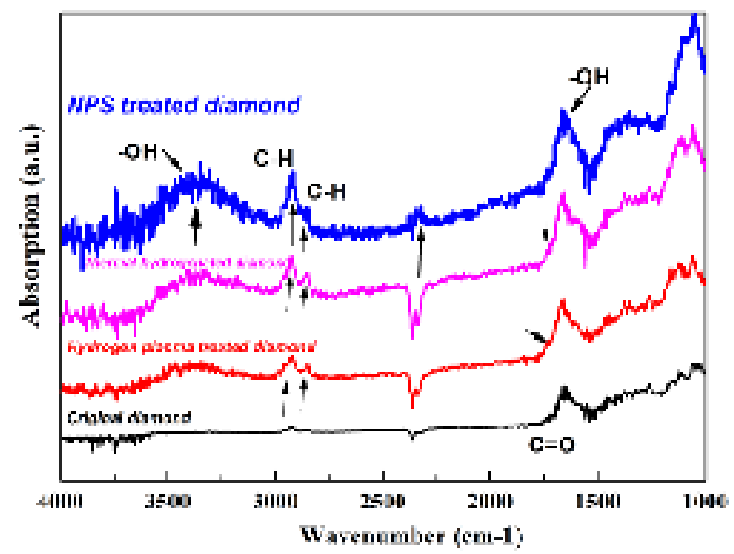

Fig.7The ATR-FTIR s pectra of the original PCD, hydrogen plasma-treated PCD,thermal hydrogenated[7]and NPS recovered FET-structure hydrogen terminatedPCD device.

ATR-FTIR spectroscopycan beused for the surface analysis of materials, including C-H bond detectionin a hydrogen-terminated diamond. Due to the similar C-H stretching vibration mode in the ATR spectra, it can be speculated that differences between the diamond crystalline orientation of both the hydrogen plasma- and NPS-treated samples were very small because they were from the same free-standing diamond wafer. Fig. 7 shows the ATR-FTIR spectra of the original DC arc jet PCD, hydrogen plasma treated PCD and NPS recovered FET-structure PCD device as well as thermal hydrogenated PCD[7], which can be acted as references to verify the C-H bonds recoveryand as an evidence of characteristic of repaired diamond device. Compared with the spectra of the original diamond, the $-\mathrm{OH}$ bonds of the NPS-treated diamond peaked at $1630 \mathrm{~cm}^{-1}$, which was related to absorbed waterand the $3400-3500 \mathrm{~cm}^{-1}$ of $-\mathrm{OH}$ bonds may have been due to the residual group or remaining moisture[28]. This is the reason why these two broad absorption peaks of the $\mathrm{SRD}$, which has been reacted in aqueous environment, were more obvious. Notably, there were $\mathrm{C}=\mathrm{O}$ peaks in the spectra of the original and hydrogen plasma-treated diamonds. This was due to the terminated oxygen or carboxyl group of the acid-washed original diamond and the remaining oxygen termination after being hydrogenated by plasma, respectively. However, in an aqueous environment, due to the surface reaction during the test in the KSS solution or surface bonding between $\mathrm{C}$ atoms and huge amounts of $\mathrm{H}^{+}$ ions under the negative potential supplied in strong acid, the $\mathrm{C}=\mathrm{O}$ bonds of the diamond surface could be broken. In addition, regarding the peak position of around $2350 \mathrm{~cm}^{-1}$, it was assigned to the carbon dioxide[29]. However, this peak was almost disappeared after NPS process, as shown in the spectrum of NPS treated diamond. Whether itcame from carbon $s p^{2}$ hybridization with tiny oxygen in vacuum chamber at high temperature of plasma condition or thermal heating? It is not very clear yet, but chemical reaction was indeed suppressed this peak. This problem still needs to be investigated deeply. Meanwhile, it wasnoted that the NPS-treated SRD sample showedmore C-H bonds, according to the absorption peak positions of $2850 \mathrm{~cm}^{-1}$ and $2930 \mathrm{~cm}^{-1}$ in Fig 7, with a higher intensity compared to the plasma-treated sample. These two absorption peaks were assignedto the $\mathrm{CH}_{2}$ symmetric stretching and $\mathrm{C}-\mathrm{H}$ bond, respectively[29]. As shown in Fig 7, although the higher density of C-H bonds of thermal hydrogenated diamond was achieved in our previous work[7],the NPS process was resulted in more $\mathrm{C}-\mathrm{H}$ bonds.It was confirmed thatthe formation of hydrogen termination on the diamond surface was due to the negative potential controlled interface reaction between the hydrogen ions and fresh carbon atoms of a diamond surface in an aqueous solution. Theoretically, for a hydrogen-terminated diamond, the more $\mathrm{C}-\mathrm{H}$ bonds that exist, the more holes that can be activated duringcontact with oxidizing substances in an aqueous environment. This means that the SRD could be more sensitive than plasma-treated PCD. C-H bonds' stability of repaired bonds should be further investigated; thus, the SRD was tested after four weeks of storage.In addition, higher proportion (intensity ratio: $I_{\mathrm{C}-\mathrm{H} / \mathrm{C}-2 \mathrm{H}}$ ) of $\mathrm{C}-\mathrm{H}$ than 
$\mathrm{CH}_{2}$ that maybe is the reason why the NPS recovered device shown more sensitivity in aqueous environment compared to plasma treated one, which will be shown in the following part.
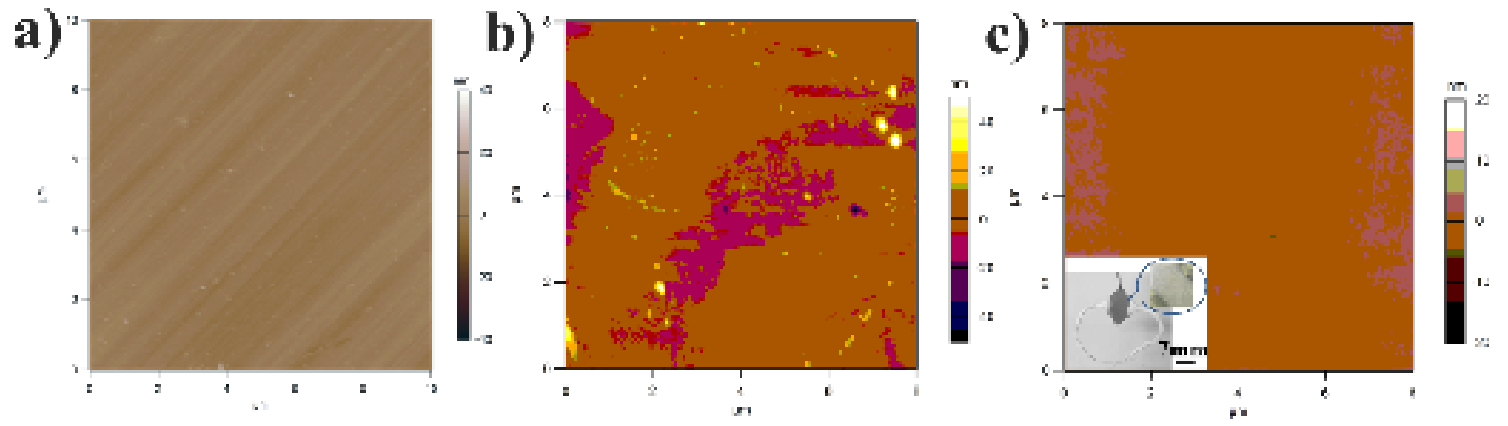

Fig. 8 S urface morphology of the PCD of the device: a) an AFM image of surface-polished PCD, b) an AFM image of a hydrogen plasma-treated PCD for the device used, and c) an AFM image andphotograph of arepaired S RD. The inset photograph is the actualFET-like structure device and it used diamond film

a)

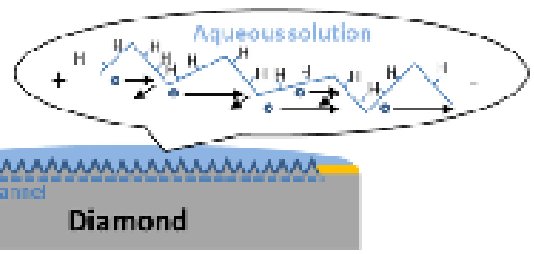

b)

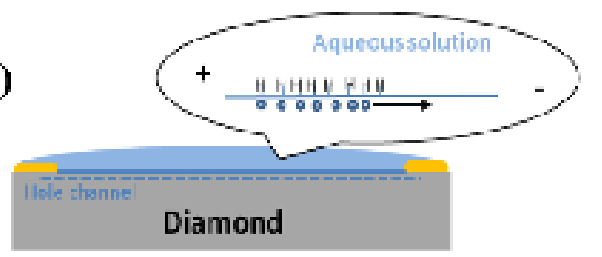

c)

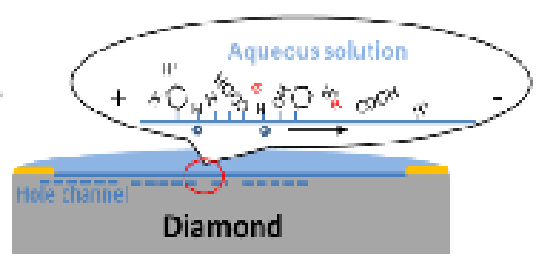

d)

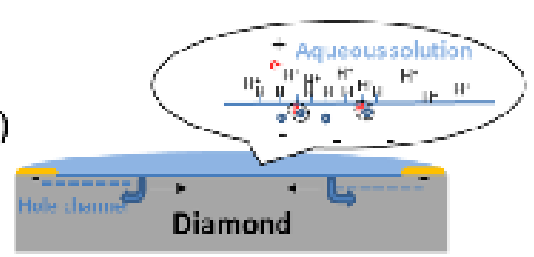

Fig.9 S chematics of relationship between surface roughness and hydrogen termination as well as mechanism of termination reaction in aqueous solution: a) surface roughness effect on hole movement, b) smooth(after polishing and hydrogenation etching) surface effect on hole movement, c) surface $\mathrm{C}-\mathrm{H}$ bonds replacement effect on hole channel, d) surface $\mathrm{C}-\mathrm{H}$ bonds recovery mechanism by NPS

Theoretically, the carrier mobility would be affected by a scattering centersuch as impurities and defects in diamond films as well as especially rough surface[7]. Fig.8 shows the surface morphology of the PCD of the deviceafter polishing, plasma hydrogenation and surface reaction in aqueous environment. As theactual foundation of graphical schematicswhichare presented in Fig.9, the actual device and it used diamond film are directly displayed in the inset photograph in Fig.8. Regarding the conductive channel on a hydrogen-terminated diamond surface, surface 
roughness scattering is one of the most important factors in limiting the carrier mobility; this is due to the two-dimensional hole gas characteristic, as graphically presented in Fig.9(a). In Fig.8(a), the diamond surface shows slight parallel scratches after mechanical polishing, and the surface roughness had an RMS of $1.78 \mathrm{~nm}$; this was done to avoid surface holes scattering and to enhance the sensitivity and conductivity of the device in an aqueous solution. Fig.8(b) shows the hydrogen plasma-treated polished PCD; the scratches have disappeared, but several tiny cavities have been created (perhapsdue to some dislocations or grain boundaries etched by the hydrogen plasma). However, the surface roughness did not increased obviously, and the RMS was just $2.13 \mathrm{~nm}$. In general, the hydrogen plasma etching process was employed to remove the surface polishing grooves before homo-epitaxial growth of high-quality diamond, e.g., high grade single crystal diamond[22]. Therefore, hydrogen termination formationprocess by plasma will result in more smoother diamond surface, of which had already been accurately polished.As is shown in the Fig.9(b), the hole movement can be more mobile without the surface roughness. Namely, the smooth surface is beneficial to enhance the carrier mobility. In addition, it should be mentioned that there were some white dots in Fig.8(a) and (b), which were dust particles in the air during testing process. After the surface reaction in a mixed solution and reparation in strong inorganic acid, which asshown in Fig.8(c) and in schematic view of Fig.9(c), the surface's physical condition (such as the roughness of a different area of SRD) was still the same, although some C-H bonds was replaced by other kinds of chemical groups. This shows that the surface reaction cannot cause any damage or synthesize new products. Also, the leakage current which is shown as electrons in Fig.9(c) graphically illustrate the reason why the curve in Fig.4(c) was fluctuated during testing. In Fig.9(d), during the process of NPS, corresponding to the Fig.5, a large amount of $\mathrm{H}^{+}$ions reached the diamond surface that resulting in the formation of $\mathrm{C}-\mathrm{H}$ bonds with $\mathrm{C}$ atoms; thisbrought about electrons transferring easily to $\mathrm{HCl}$ solution. The apparent increase and fluctuation of current during NPS process in Fig.5 indicate that surface recovery was occurred, and more electrons were able toleave the diamond surface thanks to the reformed $\mathrm{C}-\mathrm{H}$ bonds. The stability and sensitivity of repaired SRD isanother significant property for the validity of NPS and device application in aqueous environment.

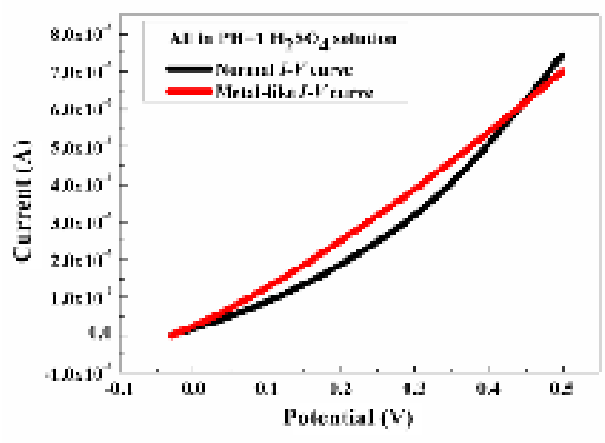

Fig. 10.I-V characteristics of the general pattern of the original hydrogen plasma-treated device and the metal-like pattern of the one-time repaired SRD by NPS in the $\mathrm{H}_{2} \mathrm{SO}_{4}$ solution.

Fig.10 shows the general curve of the original plasma-treated device and the metal-like curve of the one-time NPS-repaired SRDwhich was tested one month later in an identical solution. The metal-like curve was a straight line, and the resistance of $70.1 \mathrm{k} \Omega$ was calculated approximately according to the slope of the $I-V$ curve. The resistance of the device in aqueous solutions was generally higher than that in atmospheric environments. In the lower potential range of 0 to 0.4 , the current of the one-time NPS-repaired SRDwas higher than the original hy drogen plasma treated diamond and linearly increased (like metal). This result indicates that the repaired hydrogen terminated diamond surface by NPS was more sensitive than the hydrogen plasma-treated diamond in the solution environment. This was due to more (and higher intensity) $\mathrm{C}-\mathrm{H}$ bonds being re-formed and $\mathrm{C}=\mathrm{O}$ cleaning of the diamond surface.This may have also been due to the $\mathrm{C}-\mathrm{H}$ bonds, which formed by an NPS process in an aqueous situation; C-H bondswere 
maybemore easily activated in aqueous environments. Therefore,hydrogen terminatedPCD,which is commercially availableand providesa higher cost performance for electrochemical sensors, will achieve a better performance by NPS than plasma treatment,depending onthe development of NPS technology at a lower negative potential.

\section{Conclusion}

The super chemical inertness combined with semi-conductive characteristic caused by non-damaged surface hydrogenation has opened upa new field for the amazing application of hydrogen terminated diamond in chemical and electrochemical area. In the present work, the direct-current(DC)characteristics andthe change in resistance of a hy drogen-terminated DC arc jet plasma chemical vapor deposition (CVD) polycrystalline diamond, which has a high cost-performance, were characterized by $I-V$ experimentsbased on a field effect transistor (FET)-like structure device in multipleaqueoussolutions. The variation trends of the DC characteristic of devicein different solutions do not monotonically change based on $\mathrm{pH}$ values. The curve of current can be additionally affected by disparate ionsor organic molecules. Especially, the changing rule (which based on $\mathrm{pH}$ value) was the opposite in organic acid solutions to what it is in inorganic acid solutions. More importantly, the raised resistances of the FET-like device (from $13.57 \mathrm{k} \Omega$ to $95.78 \mathrm{k} \Omega$ ), whichwere due to the replacement of hydrogen-termination $(-\mathrm{H})$ withother organic groups which caused by surface reaction, can be reduced from $94.33 \mathrm{k} \Omega$ to $30.46 \mathrm{k} \Omega$ via NPS in a strong inorganic acid under low negative potential (-1 to $-3 \mathrm{~V}$ ). After four weeks of storage, a higher re-formed $\mathrm{C}-\mathrm{H}$ bond intensitydiamond surfaceresulted ina repaired device that was more sensitive in aqueous solution environments than the original plasma-hydrogenated device. These findings also will be the references of hydrogen termination failure and reparation of other types ofdiamond in aqueous environment.

\section{Acknowle dgements}

This work was supported by the National Key Research and Development Program of China (No.2016YFE0133200 and 2018 YFB0406500) and European Union's Horizon 2020 Research and Innovation Staff Exchange Scheme (No. 734578) and Nano-X experimental cooperation project(H008-2017).Special thanks to the national high-level university-sponsored graduate programof China Scholarship Council (CSC).

\section{References}

[1] JIsberg, JHammersberg, EJohansson,TWikstrom, DJTwitchen, AJWhitehead, SECoe, GAScarsbrook. High carrier mobility in single-crystal plasma-deposited diamond, Science., 297(2002)1670-1673.

[2]RTPiotrowska, KFabisiak, KPaprocki, MSzybowicz, EStaryga, ABanaszak. Electrochemical properties of undoped CVD diamond films,J. Phys. Chem. Solids.,72 (2011) 1225-1229.

[3]SShikata.Single crystal diamond wafers for high power electronics,Diam.Relat.Mater., 65(2016)168-175.

[4] CENebel, HKato, BRezek, DShin, DTakeuchi, HWatanabe, TYamamoto.Electrochemical properties of undoped hy drogen terminated CVD diamond, Diam.Relat.Mater., 15(2006):264 - 268.

[5] CMLi, JLLiu, LXChen, JJWei, L F Hei1, J JWang, ZH Feng, H Guo. An amazing semiconductor choicefor high-frequency FET: H-terminatedpolycry stalline diamond film prepared by DC arc jet CVD, Phys. Status. Solidi. C., 11(2014):1692-1696.

[6] F X Lu, W Tang, C M Li, J H Song, L F Hei, Application of DC arc plasma jet in the commercialization of CVD diamond films, Heat. Trea. Met., 33(1):43-48

[7] JLLiu LXChen, YTZheng, J J Wang, Z H Feng, C M Li.Carrier transport characteristics of H-terminated diamond films prepared using molecular hydrogen and atomic hydrogen, Int. J. Min. Met. Mater., 24(2017):850-856. 
[8] CIPakes, JAGarrido, HKawarada.Diamond surface conductivity: Properties, devices, and sensors,MRS.Bull., 39(2014)542-548.

[9] AHartl, JAGarrido, SNowy, RZimmermann, C Werner, D Horinek, R Netz, M Stutzmann. The Ion Sensitivity of Surface Conductive Single Crystalline Diamond,J. Am. Chem. Soc., 129 (2007)1287-1292.

[10] MTEdmonds, M Wanke, ATadich, H M Vulling, K L Rietwyk, P L Sharp, C J Stark, Y Smets, A Schenk, Q H Wu, L Ley, C I Pakes. Surface transfer doping of hydrogen-terminated diamond by C60F48: Energy level scheme and doping efficiency,J.Chem.Phys.,136(2012)124701.

[11]TTsubota, SIda, OHirabayashi, SNagaoka, M Nagata,YMatsumoto.Chemical modification of hydrogenated diamond surface using benzoyl peroxides,Phy s.Chem.Chem.PHys.,4(2002)806-811.

[12]C M Li, HLi, DNiu, F X Lu, W Z Tang, G C Chen, HZhou, FChen. Effects of residual stress distribution on the cracking of thick freestanding diamond films produced by DC arc jet plasma chemical vapor deposition operated at gas recycling mode, Surf. Coat. Tech., 201 (2007) 6553-6556

[13] KAn, L X Chen, X B Yan, X Jia, YZhao, Y T Zheng, J L Liu, JJWei, F X Lu, C M Li. Fracture behavior of diamondfilms deposited by DC arc plasma jet CVD, Ceram. Int., 44 (2018) 13402-13408

[14] S Liu, J L Liu, C M Li, J C Guo, L X Chen, JJWei L F Hei, F X Lu. The mechanical enhancement of chemical vapor deposited diamond film by plasma low-pressure/ high-temperature treatment, Carbon., 65 (2013) 365 - 370

[15] C MLi, LWang, L X Chen, Z Liu, L F Hei, F X Lu. Free-standing diamond films deposited by DC arc plasma jet on graphite substrates with a destroy able Ti interlayer, Diam. Relat. Mater., 18 (2009) 1348-1352

[16] YGurbuz, OEsame, ITekin, WPKang, JL. Davidson.Diamond semiconductor technology for RF device applications.Solid. State. Electron., 49 (2005)1055-1070.

[17] JJWang, ZZHe, CYu,XBSong,HXWang, FLin, Z H Feng.Comparison of field-effect transistors on poly crystalline and single-crystal diamonds, Diam.Relat.Mater., 7 (2016)114-117.

[18] J L Liu, C M Li, JCGuo, RHZhu, LXChen, JJWei, L f Hei, JJWang, Z h Feng, HGuo, F X Lv.Effect of atomic hydrogen bombardment on the surface conductivity of poly cry stalline diamond films, Appl. Surf. Sci., 287 (2013) 304-310

[19]https://www.iontof.com/tof-sims-5-product-version-100mm-200mm-300mm.html/

[20]C M Li, R H Zhu, J L Liu, L X Chen, G J Chao, C Y Hua, L F Hei, J J Wei, J J Wang, Z H Feng, H Guo, F X Lu. Effect of arc characteristics on the properties of large size diamond wafer prepared by DC arc plasma jet CVD. Diam. Relat. Mater., 39 (2013), 47-52.

[21]T Schuelke, T AGrotjohn. Diamond polishing, Diam. Relat. Mater., 32 (2013) 17-26

[22]CJWidmann,WMüller-Sebert, NLang, CENebel.Homo-epitaxial growth of single crystalline CVD-diamond,Diam. Relat. Mater.,64 (2016) 1-7.

[23] JRogowski. TOF-SIMS study of morphology and chemical composition of wustite-based precursor and iron catalyst for ammonia synthesis, Appl. Surf. Sci.,469(2019) 82-89.

[24]TPetit, Ljiljana Puskar. FTIR spectroscopy of nano-diamonds: Methods and interpretation, Diam. Relat. Mater.,89 (2018) 52-66

[25] OShenderova, AMPanich, SMoseenkov, SCHens, VKuznetsov, HM Vieth.Hydroxylated Detonation Nanodiamond: FTIR, XPS, and NMR Studies, J.Phys.Chem. C., 115(2011)19005-19011.

[26] HP Boehm. Surface oxides on carbon and their analysis: a critical assessment, Carbon.,40(2002)145-149.

[27] SIqbal, MSRafique, S Akhtar, N Liaqat, N Iqbal, R Ahmad. A comparative study on finding an effective root for the introduction ofhydrogen into micro-plasma during diamond growth, J. Phys. Chem. Solids.,122 (2018) 72-86.

[28] P Thongnopkun, SEkgasit. FTIR Spectra of faceted diamonds and diamond simulants, Diam. Relat. Mater.,14 
(2005) $1592-1599$.

[29] M Varga, TIzak, V Vretenar, H Kozak, J Holovsky,AArtemenko, MHulman, VSkakalova, DS Lee,A Kromka.

Diamond/carbon nanotube composites: Raman, FTIR and XPSspectroscopic studies, Carbon., 111 (2017) 54-61. 